\title{
Presencia de plaguicidas organoclorados en sangre, El Salvador
}

\author{
DOI: https://doi.org/10.5377/alerta.v3i2.9034
}

Wilfredo Beltetón Martinez ${ }^{*}$, Carlos Enrique Hernández Ávila², Elías Argueta Hidalgo ${ }^{3}$, Susana Suarez Tamayo ${ }^{4}$, Manuel Romero Placeres ${ }^{5}$.

1, 3 Laboratorio de Control de Calidad de Alimentos y Toxicología, Instituto Nacional de Salud. 2 Departamento de Gobernanza y gestión del conocimiento, Instituto Nacional de Salud.

4 Instituto Nacional de Higiene, Epidemiologia y Microbiología. Cuba.

5 Instituto de Medicina Tropical «Pedro Kouri». Cuba.

${ }^{*}$ Correspondencia

$M$ wilitin@yahoo.es

1. (D) 0000-0001-8512-7614

f ACCESO ABIERTO

Presence of organochlorine pesticides in human blood, El Salvador

Citación recomendada: Beltetón-Martínez W, Hernández-Ávila CE, ArguetaHidalgo E, Suarez-Tamayo S, Romero-Placeres M. Alerta 2020;3(2):122-128 DOI: https:// doi.org/10.5377/alerta. v3i2.9034

\section{Recibido:}

20 de diciembre de 2019

\section{Aceptado:}

7 de julio de 2020

\section{Publicado:}

17 de julio de 2020

Contribución de autoría: WBM': Conceptualización, elaboración del protocolo y del artículo, análisis de muestras. CEHÁ2: Redacción y revisión. $\mathrm{EAH}^{3}$ : Análisis de muestras y metodología. SST ${ }^{4}$ y MRP5: metodología, revisión y edición.

\section{Conflicto de intereses:}

Los autores declaran no tener conflictos de interés.

\begin{abstract}
Resumen
Introducción. Los plaguicidas organoclorados fueron utilizados en campañas de salud pública y en prácticas agrícolas. Su uso fue prohibido al detectarse residuos tóxicos en tejidos grasos de animales y personas y su persistencia en el ambiente. En El Salvador aún se utilizan, comercializan y almacenan algunos de estos plaguicidas. Objetivo. Identificar la presencia de plaguicidas organoclorados en muestras sanguíneas, almacenadas en la seroteca del Laboratorio Nacional de Salud Pública. Metodología. Estudio transversal de las muestras almacenadas en la seroteca en el periodo de enero a octubre de 2018, analizadas a través del método de cromatografía de gases con detector de microcaptura de electrones. Resultados. Se identificó presencia de plaguicidas organoclorados en 33 muestras (34\%); de las cuales 28 se detectaron en hombres. De las muestras positivas 27 eran de personas mayores de 29 años. En los departamentos de La Libertad (7) y San Salvador (6) se identificó mayor cantidad de muestras positivas a plaguicidas para el Dichloro diphenyl dichloroethane y el Lindano. Conclusiones. Se identificó presencia de algunos plaguicidas organoclorados en las muestras de la seroteca del Laboratorio Nacional de Salud Pública, lo que da indicios de la exposición de la población a plaguicidas en el medio ambiente, agua y/o alimentos.

Palabras clave

Sangre, plaguicidas, organoclorados, cromatografía de gases, El Salvador.
\end{abstract}

\section{Abstract}

Introduction. Organochlorine pesticides were widely used in public health campaigns and agricultural practices. Its use was restricted and in some cases prohibited when residues of these toxins were detected in fatty tissues of animals and people, as well as their excessive persistence in the environment. However, some of these pesticides are still in use, promoted, and stored in El Salvador. Objective. To identify the presence of organochlorine pesticides in serum samples stored in the National Laboratory of Public Health (LNSP). Methodology. A cross-sectional study was conducted over samples stored in the (NLPH) during the period from January to October 2018, which were analyzed by the gas chromatography method with electron microcapture detector ( $(\mathrm{EECD})$. Results. Organochlorine pesticides were identified in 33 samples (34\%), of which 28 were from men. Of the positive samples, 27 were from people older than 29 years. In the department of La Libertad (7), and San Salvador (6), a greater number of samples were identified positive for pesticides Dichloro diphenyl dichloroethane and lindane. Conclusions. The presence of some organochlorine pesticides was identified in the seroteca samples of the National Laboratory of Public Health, which indicates the population's exposure to pesticides in the environment, water and/or food.

\section{Keywords}

Blood, pesticides, insecticides organochlorine, chromatography gas, El Salvador 


\section{Introducción}

Desde la introducción del uso de plaguicidas en el sector agrícola y salud pública, estos han proporcionado grandes beneficios para la protección de los cultivos y para el control de vectores de enfermedades. Sin embargo, con las investigaciones realizadas hasta hoy, son evidentes los efectos indeseables de los plaguicidas sobre la salud del ser humano y sobre el medio ambiente, ya que fueron creados para interferir algún sistema biológico en particular y carecen de selectividad real. Afectan simultáneamente tanto a la especie diana como a otras categorías de seres vivos, particularmente al ser humano ${ }^{1-3}$.

Los plaguicidas organoclorados (POC) son conocidos por su alta toxicidad, lenta degradación y bioacumulación ${ }^{3}$. Se almacenan principalmente en tejidos ricos en grasa, se transportan a través de las grasas y las lipoproteínas circulantes del organismo ${ }^{4}$. La exposición a bajos niveles de POC durante períodos prolongados puede tener efectos crónicos tales como malformaciones congénitas, efectos mutagénicos, cáncer, daños en la piel, pulmones, ojos, sistema nervioso central, sistema inmunológico y esterilidad masculina $a^{5,6}$.

En El Salvador se han utilizado POC durante más de 30 años, siendo el endosulfán el único que se continúa aplicando en algunos cultivos, por lo que se hace necesario realizar un seguimiento biológico para mostrar en qué extensión dichos compuestos químicos ingresan en el ser humado desde el medioambiente. Este seguimiento refleja mejor la exposición que la determinación de POC en aire, agua, suelos y alimentos, ya que tiene en cuenta todas las rutas de exposición (ingestión, absorción dérmica, inhalación) y todas las posibles fuentes de exposición?

Este estudio tiene como objetivo identificar la presencia de plaguicidas organoclorados en muestras sanguneas, almacenadas en la seroteca del Laboratorio Nacional de Salud Pública.

\section{Metodología \\ Diseño del estudio}

Se realizó un estudio transversal descriptivo para identificar la presencia de residuos de plaguicidas organoclorados en muestras de la seroteca del LNR, procedentes de los bancos de sangre del Sistema Nacional Integrado de Salud (SNIS), en el periodo de enero a octubre de 2018.
EI LNSP cuenta con una seroteca ubicada en la sección de inmunología, las cuales se obtienen de los bancos de sangre del SNIS. Dichas muestras son referidas para realizar el control de calidad para la confirmación de agentes infecciosos, las cuales son almacenadas en caso que necesiten hacerles nuevas pruebas confirmativas u otro tipo de análisis con fines de investigación científica.

Según los registros de ingreso de muestras de la sección de bancos de sangre, en el periodo de enero a octubre de 2018 se recibieron 1288 muestras, realizando el muestreo en noviembre 2018 y el análisis en los meses de diciembre de 2018 a enero de 2019.

Las variables que se utilizaron fueron edad, sexo y lugar procedencia, las cuales se obtuvieron del formulario de control de calidad de donantes de sangre. Como lugar de procedencia se toman en cuenta los 14 departamentos de El Salvador, así como sus 262 municipios. Las variables plaguicidas organoclorados y concentración de plaguicidas en suero se obtuvieron de los análisis de laboratorio. Se analizaron y cuantificaron aldrín, dieldrín, lindano, endosulfan, diclorodifenil tricloroetano (DDT), diclorodifenil dicloroetano (DDD) y diclorodifenil dicloroetileno (DDE).

Para la elegibilidad de las muestras se designaron los siguientes criterios: se incluyeron muestras recibidas entre los meses de enero a octubre del año 2018, que tuvieran el formulario adecuadamente llenado, sin vacíos de información sociodemográfica. Se excluyeron las muestras con un volumen menor de $200 \mu \mathrm{L}$ de suero, tubos dañados, muestras duplicadas de un mismo paciente y las de donantes que no residían en El Salvador.

Para el tamaño de la muestra, se tomó un margen de error del $10 \%$ y un intervalo de confianza del $95 \%$. Debido a que no se conoce la prevalencia de los POC en muestras biológicas, se asignó una probabilidad del $50 \%$, lo que produjo un tamaño mínimo de 90 muestras de sueros para el estudio. Se realizó un muestreo aleatorio sistemático, tomando 1 muestra cada 12, recolectando hasta completar 100. De esos sueros se obtuvieron 96 muestras efectivas.

El análisis de la información se realizó en los programas Libre Office y Epidat versión 4.2. Se elaboraron tablas de frecuencias absolutas y relativas para el análisis univariado. El análisis bivariado incluye la relación entre presencia de plaguicidas con ubicación geográfica, edad y sexo. 


\section{Procesamiento de las muestras}

Para el análisis de contaminantes en sangre debe realizarse una extracción adecuada, que garantice una buena exactitud y precisión en los resultados, para lo cual se eligió utilizar la técnica de micro extracción líquido-líquido dispersiva (MELLD). Esta técnica consiste en separar los interferentes presentes en un pequeño volumen de matriz líquida, empleando un agente dispersante y en retirar los analitos, utilizando pequeños volúmenes de un agente extractante. Luego se procedió a determinar la presencia de plaguicidas organoclorados por la Técnica de Cromatografía de Gases con detector de microcaptura de electrones ${ }^{8}$.

El protocolo de investigación fue aprobado por el Comité de Ética del Instituto Nacional de Salud (INS)

\section{Resultados}

De las 96 muestras de suero 73 proceden de donantes de sangre del sexo masculino. Los rangos de edad se encuentran entre 18 a 64 años, con un promedio de 37 años y desviación estándar de 11 años. Se obtuvieron muestras de todos los departamentos a excepción de Chalatenango, equivalentes a 66 municipios, lo cual representa el $25 \%$ de la totalidad de municipios.

En la tabla 1 se muestra el porcentaje de cada plaguicida encontrado y las frecuencias en cada una de las variables en estudio.

Se logró determinar presencia de plaguicidas en 33 muestras (34,4\%), encontrando un plaguicida en 18 muestras (18,8\%) y dos plaguicidas en 15 muestras (15,6\%). No se detectó muestras con presencia de más de dos plaguicidas.

Tabla 1. Características sociodemográficas de los donantes de sangre de las muestras tomadas de la seroteca del LNSP en el año 2018, porcentaje y concentración de plaguicidas encontrados.

\begin{tabular}{lrrrrr}
\hline \multirow{2}{*}{ Variables } & \multicolumn{5}{c}{ Porcentaje de plaguicidas encontrados } \\
\cline { 2 - 5 } & $\mathrm{N}$ & \% de presencia & DDE & \multicolumn{1}{c}{ Lindano } & Dieldrín \\
\hline Sexo & 73 & $38 \%(28)$ & $32 \%(23)$ & $18 \%(13)$ & $4 \%(3)$ \\
Masculino & 23 & $22 \%(5)$ & $22 \%(5)$ & $17 \%(4)$ & 0 \\
Femenino & & & & & \\
Edad (años) & 24 & $25 \%(6)$ & $25 \%(6)$ & $21 \%(5)$ & $4 \%(2)$ \\
18 a 28 & 45 & $36 \%(16)$ & $24 \%(11)$ & $20 \%(9)$ & $4 \%(1)$ \\
29a 40 & 27 & $41 \%(11)$ & $37 \%(10)$ & $11 \%(3)$ &
\end{tabular}

\section{Concentración de plaguicidas en $\mathrm{ng} / \mathrm{mL}$}

\section{Departamento}

\begin{tabular}{|c|c|c|c|c|c|}
\hline Ahuachapán & 2 & $50 \%(1)$ & 10.7 & 7,4 & ND \\
\hline Cabañas & 4 & $50 \%(2)$ & 5.5 & ND & 10.1 \\
\hline Cuscatlán & 19 & $16 \%(3)$ & $6.9-7.8$ & $6.6-6.9$ & ND \\
\hline La Libertad & 21 & $33 \%(7)$ & $5.6-14.7$ & $6.4-7.2$ & ND \\
\hline La Paz & 8 & $50 \%(4)$ & $5.0-9.3$ & $7.3-7.6$ & 9.8 \\
\hline La Unión & 2 & $50 \%(1)$ & 5.9 & 6,7 & ND \\
\hline Morazán & 3 & $0 \%$ & ND & ND & ND \\
\hline San Miguel & 1 & $0 \%$ & ND & ND & ND \\
\hline San Salvador & 18 & $33 \%(6)$ & $5.0-18.3$ & $6.1-7.8$ & ND \\
\hline San Vicente & 5 & $60 \%(3)$ & $5.6-10.4$ & $6.5-7.2$ & ND \\
\hline Santa Ana & 2 & $50 \%(1)$ & ND & ND & 9.3 \\
\hline Sonsonate & 5 & $20 \%(1)$ & 6.6 & 5.7 & ND \\
\hline Usulután & 6 & $67 \%(4)$ & 6.3 & 11.2 & ND \\
\hline Chalatenango & 0 & $0 \%$ & ND & ND & ND \\
\hline
\end{tabular}




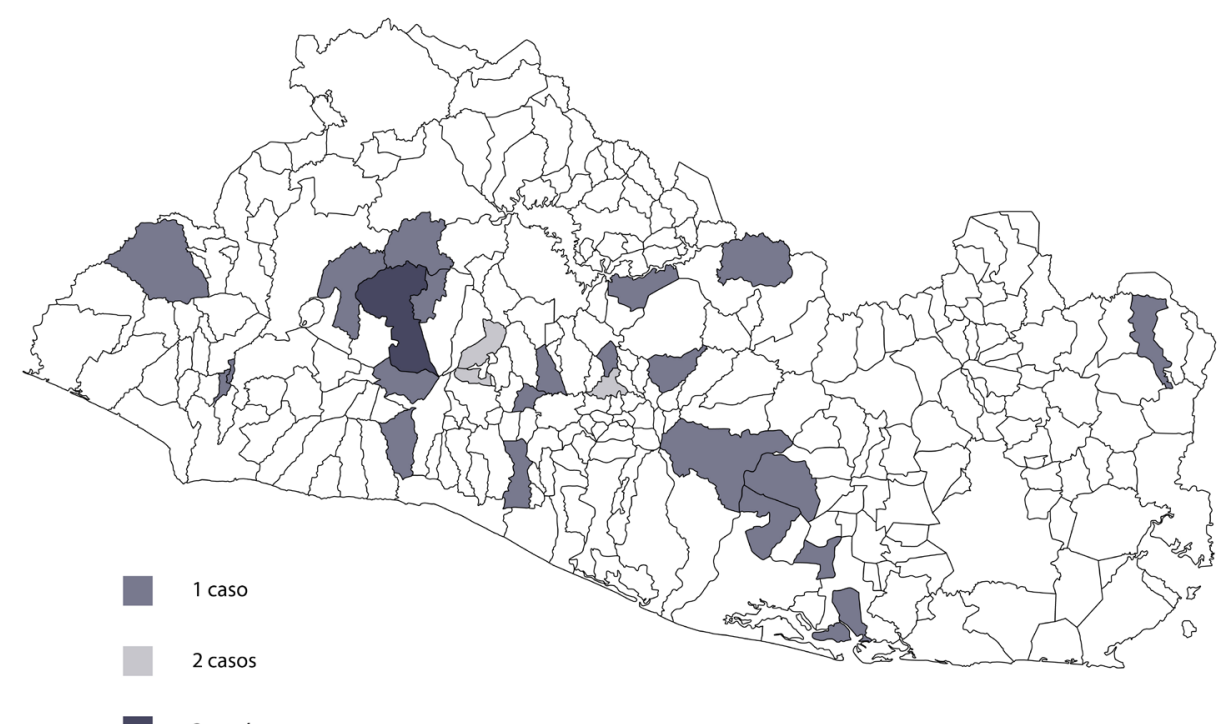

Figura 1. Frecuencia de plaguicidas organoclorados encontrados en la seroteca del LNSP en el año 2018 por municipio.

Tabla 2. Frecuencia de plaguicidas organoclorados encontrada en la seroteca el LNSP en el año 2018.

\begin{tabular}{lrrrrr}
\hline Plaguicida & Frecuencia & $\begin{array}{l}\text { Porcentaje en } \\
\text { los positivos* }\end{array}$ & $\begin{array}{l}\text { Porcentaje en } \\
\text { la población } \\
\text { total** }\end{array}$ & $\begin{array}{l}\text { Rango de } \\
\text { concentración } \\
\text { en ng/mL }\end{array}$ & $\begin{array}{l}\text { Concentración } \\
\text { promedio (ng/ } \\
\mathrm{mL})\end{array}$ \\
\hline DDE & 27 & $82 \%$ & $28 \%$ & 5,0 a 18,3 & 8,2 \\
Lindano & 17 & $51 \%$ & $18 \%$ & 5,7 a 9,2 & 7,1 \\
Dieldrín & 3 & $9 \%$ & $3 \%$ & 9,3 a 10,1 & 9,8 \\
Endosulfán 1 & 1 & $3 \%$ & $1 \%$ & 3,6 & 3,6 \\
\hline
\end{tabular}

${ }^{*} \mathrm{~N}=33$

${ }^{* *} \mathrm{~N}=96$

\section{Discusión}

Con los resultados obtenidos se confirma la presencia de DDE, lindano, endosulfán 1 y dieldrín en las muestras de sangre de la seroteca, siendo el más frecuente el DDE con 27 casos, lo cual era esperado dada su extensa vida media ${ }^{3}$ y alto potencial de bioamagnificación ${ }^{10}$.

El DDT es difícilmente metabolizado por el hombre, mientras que el resto de los seres vivos lo metabolizan de forma relativamente rápida a DDE. Por esta razón se suelen emplear los niveles de DDE como marcador de exposición crónica al DDT y se asume que el DDE presente en la población procede de fuentes exógenas9,10. Este contaminante puede provenir de exformuladoras de DDT, lugares de almacenamiento y suelo contaminado que luego pasa a las plantas y a los animales, siguiendo la cadena trófica hasta los seres humanos. El porcentaje de presencia de estos plaguicidas en la población total es comparable con un estudio realizado en Brasil en 2017 en donantes de sangre, donde se encontró una presencia de $32 \%$ de DDE, $20 \%$ de lindano y $3.7 \%$ de endosulfán $1^{11}$.

En este estudio se encontró mayor frecuencia de presencia de POC en hombres, posiblemente porque en El Salvador la mayoría de donantes de sangre son hombres como en investigaciones realizadas en Brasil y Corea ${ }^{11,13}$. Sin embargo, a pesar de que la presencia de plaguicidas se obtuvo en mayor frecuencia en los hombres, la concentración promedio en general fue mayor en las mujeres. Los niveles más altos en las mujeres pueden ser el resultado del aumento de la vida media debido a la afinidad de los POC con el contenido de grasa corporal, dado que las mujeres poseen en promedio más grasa corporal que los hombres de la misma edad"1.

Para la variable edad se observa que a medida aumenta la edad aumenta la presencia de DDE, lo cual es comparable con investigaciones realizada en Túnez, Corea, España y Rumania, donde demostraron que existe correlación entre la concentración de DDE y la edad 12,13,10,14. Esta asociación podría ser causada tanto por una bioacumulación 
dependiente de la edad como por el efecto de la cohorte de nacimiento. La primera explicación posible es que la vida media de los POC puede variar según la edad, lo que resulta en una disminución de las tasas de excreción al aumentar la edad. El efecto de la cohorte de nacimiento significa que los sujetos mayores pueden haber experimentado en el pasado una mayor exposición que las personas más jóvenes, como se observó en otros países ${ }^{11}$.

Caso contrario los casos positivos a lindano se obtuvieron en un $76 \%$ de las personas menores de 40 años, sugiriendo que es debido a que en El Salvador el lindano fue prohibido hasta en el año 2011 y su principal uso era medicinal para combatir los ectoparásitos en niños.

El endosulfán 1 solo se encontró en una muestra, a pesar de que aún se comercializa en El Salvador. Cabe mencionar que en el año 2004 se prohibió su uso para aplicaciones aéreas y a menos de veinte metros de las fuentes de agua. Además, desde el 2014 el Ministerio de Agricultura y Ganadería (MAG) está impartiendo capacitaciones para la implementación de las buenas prácticas agrícolas como alternativa al endosulfán para el control de plagas, lo que podría favorecer a que se esté disminuyendo el uso de dicho plaguicida.

El dieldrín se encontró en tres muestras de sexo masculino de 34, 37 y 59 años, procedentes de Cabañas, La Paz y Santa Ana respectivamente. El dieldrín se utilizaba principalmente para proteger madera y estructuras de madera contra el ataque de insectos y termitas y en la industria para combatir plagas textiles ${ }^{16}$.

No se encontraron DDT, DDD, aldrín y endosulfán 2, lo que se puede deber a diversos factores como por ejemplo los sujetos estudiados, el tamaño de la muestra y la degradación de los plaguicidas.

Investigaciones recientes, sugieren que los niveles de exposición que se observan en la población general podrían estar relacionados con diversos efectos en la salud, especialmente en los efectos metabólicos y enfermedades crónicas como la diabetes tipo $2^{15}$, la enfermedad renal crónica de causas no tradicionales en El Salvador ${ }^{17}$, linfoma no Hodgkin y cáncer testicular y cáncer de seno ${ }^{18}$.

Según un estudio sobre la epidemiología de las intoxicaciones agudas por plaguicidas en El Salvador, se encontró que entre 2012 y 2015 hubo 110 intoxicaciones por POC, de las cuales se declararon 26 por suicidio, 48 laborales, 35 accidentales y 1 por homicidio. De estas intoxicaciones 60 fueron causadas por el endosulfán, por lo que las otras 50 fueron causadas por POC prohibidos ${ }^{19}$.

De los 13 departamentos de los que se obtuvieron muestras, en 11 se detectó presencia de plaguicidas organoclorados.

Los resultados de este estudio no pueden ser generalizarlos a la población adulta de El Salvador, dado que los donantes de sangre son individuos más saludables que la población general. A pesar de esto, los donantes de sangre representan una valiosa oportunidad para realizar un seguimiento biológico de exposición a productos químicos en una población específica debido a la practicidad de la recolección de muestras de sangre y su buena predisposición a colaborar. No es posible comparar la distribución de los plaguicidas con estudios previos en el país, pero en virtud de lo anterior es probable que la población se encuentre expuesta indirectamente a estos plaguicidas.

La principal fuente de exposición es la alimenticia, debido a que los $P O C$ se van acumulando en el organismo por la biomagnificación; proceso mediante el cual, el plaguicida se incorpora a la cadena trófica hasta llegar al ser humano, corriendo el riesgo de absorber las sustancias contaminantes magnificadas. Debido a que no existe una normativa que establezca los niveles máximos permisibles en sangre humana, no es posible establecer si estos resultados son perjudiciales a la salud, pero la presencia de estos compuestos que no deberían estar presentes en el torrente sanguíneo, puede ser un factor de riesgo por varios problemas de salud.

La prohibición de los POC Ilevó a una disminución esencial en la carga corporal de estos tóxicos en la población general, lo que se refleja en la baja concentración en individuos más jóvenes. Sin embargo, las concentraciones circulantes de POC, incluso a niveles como los encontrados en este estudio, siguen siendo motivo de preocupación; en primer lugar, porque no debería existir presencia de dichos compuestos en la sangre de la población. En segundo lugar, no se conoce la ocupación de las personas de las que proceden los sueros en estudio, que podría indicar si la presencia de los POC es por exposición directa o indirecta.

Dados los resultados de este estudio se comprueba que en El Salvador existe exposición crónica a los POC. Esto es de gran importancia para la salud pública debido a que la presencia de residuos de estos compuestos en fluidos corporales y tejidos puede ser un factor de riesgo para varios problemas de salud y despierta una especial preocupación que se detectaron niveles de plaguicidas en sujetos aparentemente sanos. 
Con esto se demuestra la utilidad del seguimiento biológico para determinar la exposición a POC, por lo que se debe profundizar las investigaciones para la evaluación de riesgos toxicológicos. Esto permitiría en estudios posteriores identificar la ruta y fuentes de los contaminantes, para poder realizar acciones para prevenir daños a la salud.

\section{Conclusiones}

Existe presencia de plaguicidas organoclorados ( $\mathrm{DDE}$, lindano, dieldrín y endosulfán 1) en las muestras de suero de la seroteca del Laboratorio Nacional de Salud Pública, comprobando que los POC aún persisten en el ambiente del territorio de El Salvador pese a que se han dejado de utilizar desde hace casi 40 años aproximadamente.

A medida que aumenta la edad de la persona, mayor es la probabilidad de encontrar presencia de plaguicidas en las muestras de la seroteca del LNSP.

Existe mayor frecuencia de presencia de POC en muestras procedentes de hombres, pero una concentración promedio más alta en muestras procedentes de mujeres.

En los departamentos de La Libertad y San Salvador se encontró mayor frecuencia de presencia de POC, pero a nivel de zonas fue mayor la presencia en la zona central y paracentral de El Salvador.

\section{Agradecimientos}

Al Instituto Nacional de Salud y al Laboratorio Nacional de Salud Pública del Ministerio de Salud para la realización de los análisis respectivos y al área de bancos de sangre por brindar las muestras para ser analizadas. Al Instituto Nacional de Higiene, Epidemiologia y Microbiología y al Instituto de Medicina Tropical "Pedro Kouri" de Cuba por su colaboración en la redacción del trabajo de investigación.

\section{Referencias bibliográficas}

1. Ramírez J, Lacasaña Y. Plaguicidas: clasificación, uso, toxicología y medición de la exposición. Arch Prev Riesgos Labor. 2001;4(2):67-75. Disponible en: https:// pesquisa.bvsalud.org/portal/resource/pt/ ibc-20218.

2. Schaaf AA. Valoración de impacto ambiental por uso de pesticidas en la región agrícola del centro de la provincia de Santa $\mathrm{Fe}$, Argentina. Rev. Mex. Cienc. Agríc. 2016;7(6):1237-1247. Disponible en: http:// www.scielo.org. $\mathrm{mx} /$ scielo.php? pid=S2007$09342016000601237 \&$ script=sci arttext

3. Jayaraj R, Megha P, Sreedev P. Review Article. Organochlorine pesticides, their toxic effects on living organisms and their fate in the environment. Interdisciplinary Toxicology. 2016;9(3-4):90-100. DOl: $10.1515 /$ intox-2016-0012

4. Waliszewski SM, Caba M, Gómez S, et al. Niveles de Plaguicidas Organoclorados en Habitantes de México. Revista Internacional de Contaminación Ambiental. 2013;(29):121-

131. Disponible en: https://www.revistascca. unam.mx/rica/index.php/rica/article/ view/41613

5. Arbeláez MP, Henao H, Samuel. Vigilancia sanitaria de plaguicidas: experiencia de PLAGSALUD en Centroamérica. 2004.

Disponible en: http://cidbimena.desastres. $\underline{\mathrm{hn} / \text { filemgmt/files/RA VigilanciaSanitaria. }}$ pdf.

6. Reyes Osses RA. Determinación de plaguicidas organoclorados en suero sanguíneo de mujeres gestantes del Hospital de Lanco por Cromatografía de Gases Acoplada a Espectrometría de Masas con Ionización Química Negativa. 2011. http://cybertesis.uach.cl/tesis/uach/2011/ fcr457d/doc/fcr457d.pdf

7. Gómez DG. Espectrometría de masas en tándem acoplada a cromatografía líquida con pretratamiento online: Determinación de xenobióticos en alimentos $Y$ biomarcadores en muestras biológicas. Tesis doctoral. España, Universidad de Salamanca. 2013.462p. DOI: 10.14201/gredos.122991

8. Beltetón W, Argueta J. Implementación de metodología para la determinación de plaguicidas organoclorados en sangre humana. Revista ALERTA. 2019;2(1):66-70. DOI: $\underline{10.5377 / \text { alerta.v2i1.7537 }}$

9. Glynn AW, Granath F, Aune M, et al. Organochlorines in Swedish women: determinants of serum concentrations. Environmental Health Perspectives. 2003;111(3):349-355. DOI:10.1289/ehp.5456

10. Zumbado M, Goethals M, Álvarez EE, et al. Exposición inadvertida a plaguicidas organoclorados (DDT y DDE) en la población de las Islas Canarias. Ecosistemas. 2004;13(3):9. Disponible en https://www. redalyc.org/articulo.oa?id=54013309

11. Freire C, Koifman RJ, Koifman S. Serum levels of organochlorine pesticides in blood donors: A biomonitoring survey in the North of Brazil, 2010-2011. Science of The Total Environment. 2017;598:722-732. DOI: 10.1016/j.scitotenv.2017.04.128

12. Ben Hassine $S$, Hammami B, Ben Ameur $W$, et al. Concentrations of organochlorine pesticides and polychlorinated biphenyls in human serum and their relation with age, gender, and BMI for the general population 
of Bizerte, Tunisia. Environ Sci Pollut Res. 2014;21(10):6303-6313. DOI: $10.1007 /$

s11356-013-1480-9

13. Kim J-T, Kang J-H, Chang Y-S, Lee D-H, Choi S-D. Determinants of serum organochlorine pesticide and polychlorinated biphenyl levels in middle-aged Korean adults. Environ Sci Pollut Res. 2018;25(1):249-259. DOI: $10.1007 /$ s11356-017-0382-7

14. Dirtu AC, Cernat R, Dragan D, et al. Organohalogenated pollutants in human serum from lassy, Romania and their relation with age and gender. Environment International. 2006;32(6):797-803. DOI: 10.1016/j.envint.2006.04.002

15. Salihovic S, Ganna A, Fall T, Broeckling CD, Prenni JE, van Bavel B, et al. The metabolic fingerprint of $p, p^{\prime}-D D E$ and $H C B$ exposure in humans. Environ Int. 2016;88:60-66. doi: 10.1016/j.envint.2015.12.015.

16. Agencia para Sustancias Tóxicas y el Registro de Enfermedades. (ATSDR). Reseña Toxicológica de el Aldrín y el Dieldrín (en inglés). Atlanta, GA: Departamento de Salud y Servicios Humanos de EE. UU., Servicio de Salud Pública. 2002. Disponible en: https:// www.atsdr.cdc.gov/es/phs/es phs1.html

17. Orantes $C M$, Herrera R, Almaguer $M$, Brizuela EG, Núñez L, Alvarado NP et al. Epidemiology of chronic kidney disease in adults of Salvadoran agricultural communities. MEDICC. 2014.16(2):23-30. Disponible en: https://pubmed.ncbi.nlm. nih.gov/24878646/

18. International Agency for Research on Cancer. IARC Monographs evaluate DDT, lindane, and 2,4-D. Francia. World Heatlh Organization. 2015. 2 p. Disponible en: https://www.iarc.fr/wp-content/ uploads/2018/07/pr236 E.pdf

19. Quinteros E, López A. Epidemiología de las intoxicaciones agudas por plaguicidas en El Salvador. Revista ALERTA. 2019; 2(2). DOI: https://doi.org/10.5377/alerta. v2i2.7846 